\title{
Interaction of equatorial waves through resonance with the diurnal cycle of tropical heating
}

\author{
By CARLOS F. M. RAUPP* and PEDRO L. SILVA DIAS, Department of Atmospheric Sciences, \\ Institute of Astronomy, Geophysics and Atmospheric Sciences, University of Sao Paulo, São Paulo/SP, Brazil
}

(Manuscript received 5 August 2009; in final form 27 April 2010)

\begin{abstract}
In this work, a new theoretical mechanism is presented in which equatorial Rossby and inertio-gravity wave modes may interact with each other through resonance with the diurnal cycle of tropical deep convection. We have adopted the two-layer incompressible equatorial primitive equations forced by a parametric heating that roughly represents deep convection activity in the tropical atmosphere. The heat source was parametrized in the simplest way according to the hypothesis that it is proportional to the lower-troposphere moisture convergence, with the background moisture state function mimicking the structure of the ITCZ. In this context, we have investigated the possibility of resonant interaction between equatorially trapped Rossby and inertio-gravity modes through the diurnal cycle of the background moisture state function. The reduced dynamics of a single resonant duo shows that when this diurnal variation is considered, a Rossby wave mode can undergo significant amplitude modulations when interacting with an inertio-gravity wave mode, which is not possible in the context of the resonant triad non-linear interaction. Therefore, the results suggest that the diurnal variation of the ITCZ can be a possible dynamical mechanism that leads the Rossby waves to be significantly affected by high frequency modes.
\end{abstract}

\section{Introduction}

It is well known that the large-scale atmospheric flow has basically two different kinds of wave motions, namely the lowfrequency Rossby wave which is characterized by an approximate geostrophic balance and the high-frequency inertio-gravity or Poincaré wave, apart from the mixed Rossby-gravity (Yanai) and Kelvin waves, which are exclusively characteristic of the equatorial wave-guide (Matsuno, 1966). Due to the large gap in the time-frequency spectrum between the Rossby and Poincaré waves and the fact that most part of the energy of the large-scale motions in middle and high latitudes is in quasi-geostrophic modes, many initialization methods have been considered in numerical studies on global models of primitive equations in order to filter out inertio-gravity oscillations of unrealistic high amplitudes (Charney, 1955; Phillips, 1960; Baer and Tribbia, 1977; Kasahara, 1982; Tribbia, 1984). Initialization schemes have also been analysed in the context of the equatorial waveguide by separating the solution into slow and fast manifolds based on the time-frequency of the linear eigenmodes (Tribbia, 1979; Silva

\footnotetext{
*Corresponding author.

Institute for Theoretical Physics, UNESP - Sao Paulo State University, P.O. Box 70532-2, São Paulo, SP, 01156-970, Brazil.

e-mail: raupp@ift.unesp.br

DOI: $10.1111 / j .1600-0870.2010 .00463 . x$
}

Dias et al., 1983). An important point regarding any filtering procedure is on whether or not one loses information on the systems' dynamics with the filtering process. This point is closely related to the following specific question: can a slow (Rossby) mode interact with a fast (inertio-gravity) wave in such a way that the slow mode be significantly affected by the propagating fast wave?

To answer this question, one needs to account for the wave interaction mechanisms. As regards the non-linear wave interaction, it is well known (Bretherton, 1964; Ripa, 1981, 1982, 1983a,b; Medvedev and Zeitlin, 2005; Reznik and Zeitlin, 2007) that in the finite amplitude limit dispersive waves only interact effectively if they are resonant, that is, when they form threeor four-wave interactions with other modes of the system such that the wavenumbers and frequencies of the modes both add to zero. In this sense, any dispersive system having quadratic non-linearities to lowest order may exhibit triad resonance phenomena as leading-order non-linear effects. A rich compendium on the three-wave resonance phenomenon in fluid dynamics can be found in chapter 5 of Craik (1985). With regard to the resonant triad interaction involving Rossby and inertio-gravity waves, Duffy (1974) studied this kind of interaction in the context of the mid-latitude $\beta$-plane shallow-water equations, while Domaracki and Loesch (1977), Loesch and Deininger (1979), Ripa (1983a,b) and Raupp and Silva Dias (2006) analysed the dynamics of equatorial wave resonant triads in the equatorial 
$\beta$-plane shallow-water model and Raupp et al. (2008) extended these studies for a fully stratified model represented by the equatorial $\beta$-plane primitive equations. Medvedev and Zeitlin (2005) investigated the resonant interactions of short equatorial waves by applying the wave turbulence approach into the equatorial $\beta$-plane shallow-water equations and obtained stationary powerlaw energy spectra for the equatorial inertio-gravity waves. A fundamental result of these studies is that the non-linear coupling coefficients are in general proportional to the individual time-frequencies in the resonant triads, so that the wave having the maximum absolute time-frequency in a resonant triad always acts as an energy source (or sink) for the remaining triad components, as usually occurs with the three-wave resonances in several other problems in fluid dynamics (see chapter 5 of Craik, 1985, and references therein).

Thus, given the resonance conditions for a triad interaction to take place, the only way for a Rossby mode to interact with fast waves is to resonate with two inertio-gravity waves with nearly equal or opposite time-frequencies. Furthermore, due to the energy constraints imposed by the triad dynamics, in this sort of resonant interaction the Rossby mode essentially acts as a catalyst component for the energy exchanges between the two high-frequency modes, in the sense that it enables the resonance conditions to be satisfied and controls the interaction period through its amplitude, but its amplitude (energy) is not significantly affected by the fast propagating waves. This property explains the non-linear Rossby adjustment in mid-latitudes even for general unbalanced initial data in the limit of strong rotation (for more details on this, see Majda and Embid, 1998).

Nevertheless, apart from the non-linear wave interaction mechanism, waves in general can also interact through inhomogeneities in the medium in which they are embedded, such as a shearing basic current (Milewski, 1995; Milewski and Benney, 1995) and a varying topography (Milewski, 1998). In the atmospheric context, this interaction mechanism among waves through variation in the medium has been studied by Majda et al. (1999) in the framework of the equatorial $\beta$-plane shallow-water equations with a varying topography. Majda et al. (1999) analysed the role of a smooth varying topography in resonantly coupling equatorial waves, focusing on the interaction involving the non-dispersive Kelvin wave and a set of dispersive modes. They show that by this resonance mechanism the Kelvin wave can excite large-scale Yanai and Rossby waves with specific zonal wavelengths and slow down its propagation speed, along with preventing its breaking. In the present paper we extend the study of Majda et al. by analysing another inhomogeneous mechanism that can lead equatorial atmospheric waves to interact and exchange energy with each other through resonance. This mechanism is based upon the time and space variations of the thermal forcing associated with moist deep convection in the tropical atmosphere.

Therefore, in this work a new theoretical mechanism of interaction among equatorial atmospheric waves is presented in which equatorial Rossby and Poincaré modes can interact with each other through latitudinal and time inhomogeneities of the background distribution of moisture in the tropical atmosphere. We have adopted the two-layer incompressible equatorial primitive equations forced by a parametric heating that roughly represents deep convection in the tropical atmosphere. The heat source was parametrized in the simplest way according to the hypothesis that it is proportional to the lower-troposphere moisture convergence, with the background moisture state function being prescribed to mimic the structure of the intertropical convergence zone (ITCZ). We have particularly explored the possible resonances between equatorial Rossby and Poincaré wave modes associated with the diurnal cycle of the background moisture state function. It was found that, unlike the resonant triad nonlinear interaction, in this linear interaction mechanism through resonance with the diurnal cycle of the background specific humidity, a Rossby mode undergoes expressive energy (amplitude) modulations when interacting with an inertio-gravity mode. In order to illustrate in a simplified fashion on how this resonance mechanism works in the full dynamics, where it may compete with the three-wave non-linear resonance, we have also analysed the dynamics of a resonant non-linear wave triad composed of two equatorial inertio-gravity waves and one barotropic wave mode in which one of the inertio-gravity modes interact with an equatorial Rossby mode through resonance with the diurnal cycle of the background ITCZ function.

The reminder of this paper is organized as follows. In Section 2, we present the model equations, the heat forcing parametrization adopted and the possible resonances involving equatorial Rossby and inertio-gravity wave modes through the diurnal forcing. Section 2 also analyses the dynamics of these resonant interactions in terms of the location and width of the ITCZ. In this section, we also augment the two-wave interaction theory to include the three-wave non-linear interaction of equatorial inertio-gravity wave modes and a barotropic mode. The main conclusions of this paper and the main implications of the results for the atmospheric science are discussed in Section 3.

\section{Resonant interactions}

\subsection{Model equations and free linear wave solutions}

The original model adopted in the present work is described by the incompressible equatorial $\beta$-plane primitive equations with a constant static stability parameter of the background atmosphere given by its typical tropospheric value of $N=10^{-2} \mathrm{~s}^{-1}$. The assumption of rigid lid vertical boundary conditions where the vertical velocity $w$ vanishes at the bottom $(z=0)$ and at a hypothetical top of height $z=\mathrm{H}$ of the troposphere allows the existence of a set of discrete vertical eigenmodes that characterizes the vertical structure of the solution. The model is forced by a deep convection (first baroclinic) heat source that has been defined to roughly represent the typical deep convection activity 
in the tropical atmosphere. Under the circumstances described above, we consider the two-layer approximation of the referred model, which may be described in terms of the barotropic and baroclinic components of the horizontal velocity and pressure fields as follows:

$\frac{\partial V_{0}}{\partial t}+y V_{0}^{\perp}+\nabla p_{0}=-V_{1} \cdot \nabla V_{1}-V_{1} \operatorname{div} V_{1}$

$\operatorname{div} V_{0}=0$

$\frac{\partial V_{1}}{\partial t}+y V_{1}^{\perp}+\nabla p_{1}=-V_{0} \cdot \nabla V_{1}-V_{1} \cdot \nabla V_{0}$

$\frac{\partial p_{1}}{\partial t}+\operatorname{div} V_{1}=-\frac{\mathrm{g} \pi^{2}}{2 H^{2} N^{2} c_{p} T_{0}} S_{1}-V_{0} \cdot \nabla p_{1}$.

In (1) and (2), $V_{0}=\left(u_{0}, v_{0}\right)^{T}$ and $V_{1}=\left(u_{1}, v_{1}\right)^{T}$ refer to the barotropic and baroclinic components of the horizontal velocity field, respectively, while $p_{0}$ and $p_{1}$ represent the barotropic and baroclinic contributions, respectively, for the pressure field normalized by a reference value of the background density; $g \approx$ $9.81 \mathrm{~ms}^{-2}$ is the gravity acceleration, $c_{p}=1004 \mathrm{JKg}^{-1} \mathrm{~K}^{-1}$ refers to the thermal capacity of dry air at constant pressure and $T_{0}$ is a reference temperature and will be fixed with its standard value of $T_{0}=288.16 \mathrm{~K}\left(15^{\circ} \mathrm{C}\right)$. Equations (1) and (2) have been non-dimensionalized by using the equatorial Rossby radius $L_{E}=\sqrt{\frac{c}{\beta}}$ as unit of length and $T_{E}=(c \beta)^{-\frac{1}{2}}$ as unit of time, where $c=\mathrm{NH} / \pi$ is the characteristic speed of the linear waves and $\beta$ is the gradient of the Coriolis parameter at the equator and is assumed here to be a constant. The variables $V_{j}$ and $p_{j}, j=$ 0,1 , are non-dimensionalized by $c$ and $c^{2}$, respectively. In the equations above, $\nabla=\left(\partial_{x}, \partial_{y}\right)^{T}$ refers to the horizontal gradient operator and $S_{1}$ corresponds to the projection of the vertical derivative of the heat source onto the first baroclinic mode. The parametrization of $S_{1}$ in terms of the dynamical variables of the present model will be discussed later. Hereafter we assume the fixed values of the Brunt-Vaisala frequency $N=10^{-2} \mathrm{~s}^{-1}$ and the top height of the troposphere $H=16 \mathrm{Km}$, resulting in a gravity wave reference speed of approximately $c=51 \mathrm{~m} \mathrm{~s}^{-1}$ and in the units of length and time of $L_{E} \approx 1500 \mathrm{Km}$ and $T_{E} \approx 8.3$ $\mathrm{h}$, respectively. Equations (1) and (2) are obtained by a Galerkin truncation of the original model equations of the form

$V(x, y, z, t)=V_{0}(x, y, t)+V_{1}(x, y, t) \cos \left(\frac{\pi}{H} z\right)$

$p(x, y, z, t)=p_{0}(x, y, t)+p_{1}(x, y, t) \cos \left(\frac{\pi}{H} z\right)$

$S(x, y, z, t)=S_{1}(x, y, t) \frac{\pi}{2 H} \sin \left(\frac{\pi}{H} z\right)$.

Due to the divergenceless condition (1b), eq. (1a) for the barotropic mode may be written in terms of the stream func- tion $\psi$ according to

$$
\begin{aligned}
\frac{\partial \nabla^{2} \psi}{\partial t}+\frac{\partial \psi}{\partial x}= & -\left[V_{1} \cdot \nabla \operatorname{curl} V_{1}+2 \operatorname{curl} V_{1} \operatorname{div} V_{1}\right. \\
& \left.-V_{1}^{\perp} \cdot \nabla \operatorname{div} V_{1}\right]
\end{aligned}
$$

where the stream function $\psi$ satisfies the relations $u_{0}=-\psi_{y}$ and $v_{0}=\psi_{x}$ and $\nabla^{2}=\frac{\partial^{2}}{\partial x^{2}}+\frac{\partial^{2}}{\partial y^{2}}$ corresponds to the Laplacian operator. In the eqs (1a) and (4), we have neglected the advection term $V_{0} \cdot \nabla V_{0}$ that yields the barotropic mode self-interactions, since this kind of interaction is beyond the scope of this paper.

The linearized and unforced version of (2) has equatorially trapped linear wave eigenmodes of the form (Matsuno, 1966)

$\left[\begin{array}{l}u_{1} \\ v_{1} \\ p_{1}\end{array}\right]=\left[\begin{array}{c}u_{a}(y) \\ v_{a}(y) \\ p_{a}(y)\end{array}\right] \mathrm{e}^{\mathrm{i} k x+\mathrm{i} \omega_{a} t}$,

with the zonal wavenumber $k$ and the eigenfrequency $\omega_{a}$ satisfying the dispersion relation

$\omega_{a}^{2}-k^{2}+\frac{k}{\omega_{a}}=2 n+1$,

for $n=-1,0,1,2, \ldots$ The solutions of (5b) are displayed in Fig. 1 and correspond to the familiar equatorial wave types: mixed Rossby-gravity waves (MRGW), inertio-gravity (Poincaré) waves propagating westward (WGW) and eastward (EGW), Rossby waves (RW) and the non-dispersive Kelvin wave $(\mathrm{KW})$. The important point here is that there exists a large timescale separation between the Poincaré and the Rossby waves. For the dispersive modes (RW, WGW, EGW and MRGW), the meridional structure eigenfunctions are given by

$$
\begin{aligned}
& {\left[\begin{array}{l}
u_{a}(y) \\
\mathrm{v}_{a}(y) \\
p_{a}(y)
\end{array}\right]} \\
& =\left[\begin{array}{c}
\frac{1}{2}\left(\omega_{a}-k\right) H_{n+1}(y)+n(n+1)\left(\omega_{a}+k\right) H_{n-1}(y) \\
\mathrm{i}\left(\omega_{a}^{2}-k^{2}\right) H_{n}(y) \\
\frac{1}{2}\left(\omega_{a}-k\right) H_{n+1}(y)-n(n+1)\left(\omega_{a}+k\right) H_{n-1}(y)
\end{array}\right] \\
& \quad \times \frac{\mathrm{e}^{-\frac{y^{2}}{2}}}{\sqrt{E_{a}}} .
\end{aligned}
$$

In (5c) above, $E_{a}=2^{n} n ! \pi^{1 / 2}\left[\left(\omega_{a}-k\right)^{2}(n+1)+n\left(\omega_{a}+k\right)^{2}+\right.$ $\left(\omega_{a}^{2}-k^{2}\right)^{2}$ ] refers to the $L_{2}$ norm of the meridional structure eigenfunctions and $H_{n}(y)$ corresponds to the $n$th degree Hermite polynomial. The meridional structure eigenfunctions for the non-dispersive Kelvin mode are given by $u_{a}(y)=p_{a}(y)=$ $\frac{\mathrm{e}^{-\frac{y^{2}}{2}}}{(2 \sqrt{\pi})^{\frac{1}{2}}}$ and $v_{a}(y) \equiv 0$.

Thus, the subscript $a$ in (5), which represents a particular mode, is characterized by a zonal wavenumber $k$, the meridional index $n$ distinguishing the meridional structure of the equatorial waves and the wave type represented by $r[a=(k, n, r)]$. For 
Fig. 1. Dispersion curves of the possible equatorial wave types: Rossby waves (RW), inertio-gravity waves propagating westward (WGW) and eastward (EGW), mixed Rossby-gravity waves (MRGW) and Kelvin waves (KW).

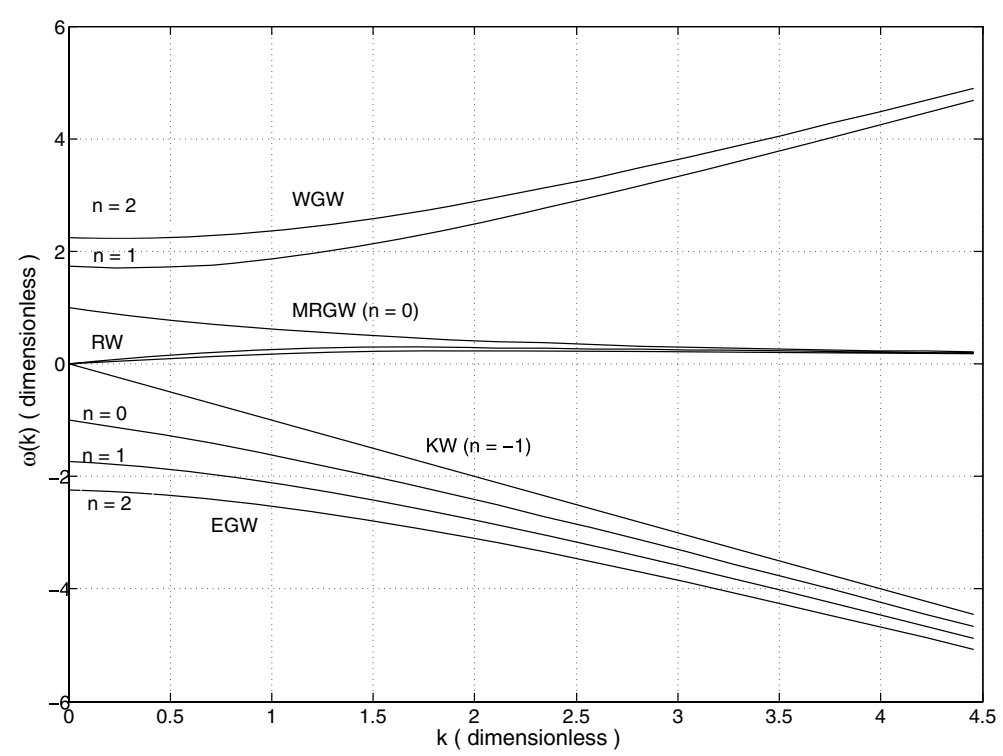

periodic boundary conditions in $x$, the zonal wavenumbers $k$ are quantized and are given by

$k=\frac{2 \pi}{L_{x}} \alpha, \quad \alpha=0, \pm 1, \pm 2, \pm 3, \ldots$

with $L_{x}$ representing the equatorial perimeter of the circumference of the earth in dimensionless units, that is, $L_{x} L_{E} \approx$ $40000 \mathrm{Km}$.

The eigensolutions of the linearized version of (4) are the barotropic Rossby waves that are no longer trapped near the equator

$\psi_{k, l}=\mathrm{e}^{\mathrm{i} k x+\mathrm{i} \omega_{B} t} \sin (l y)$

with the dispersion relation

$\omega_{B}=\frac{k}{k^{2}+l^{2}}$,

for any meridional wavenumber 1 .

\subsection{Forcing parametrization and resonances between equatorial baroclinic Rossby and Poincaré waves}

Regarding the parametrization of the heat source $S_{1}$ in (2b), we have considered the wave-CISK hypothesis in which the heating is assumed to be proportional to the lower-troposphere moisture convergence (Hayashi, 1970; Lindzen, 1974; Stevens and Lindzen, 1978; Chang and Lim, 1988)

$S_{1}(x, y, t)=-L_{c} Q(y, t) \operatorname{div} V_{1} \int_{0}^{z_{m}} \cos \left(\frac{\pi}{\mathrm{H}} z\right) \mathrm{d} z$,

where $Q(y, t)$ corresponds the background state of the specific humidity or, as will be called hereafter, the background moisture state function or ITCZ function, $L_{c}=2.5 \times 10^{6} \mathrm{JKg}^{-1}$ is the latent heat of condensation and $z_{m}$ in the integral in (8) refers to the height of the lift condensation level or the clouds' base.
Substituting (8) into (2b) yields

$\frac{\partial p_{1}}{\partial t}+\operatorname{div} V_{1}=\Gamma Q \operatorname{div} V_{1}-V_{0} \cdot \nabla p_{1}$

where

$\Gamma=g \frac{\pi L_{c}}{2 H N^{2} c_{p} T_{0}} \sin \left(\frac{\pi}{H} z_{m}\right)$.

The background moisture state function $Q(y, t)$ is prescribed to roughly represent the structure of the ITCZ, which defines the moist region of the globe where deep convection is most likely to occur in a climatological view. In this way, the function $Q(y, t)$ is defined as

$Q(y, t)=Q_{0} \exp \left[-\left(\frac{y-y_{0}}{r_{y}}\right)^{2}\right] f(t)$,

where $Q_{0}$ is the maximum value of the specific humidity and controls the magnitude of the heat forcing. In the calculations displayed in this paper we have considered its standard value of $Q_{0}=10 \mathrm{~g} \mathrm{Kg}^{-1}$. In (10), $y_{0}$ refers to the latitude of maximum deep convection activity and can assume corresponding values from 0 to $15^{\circ}$ (south and north) according to the seasonal cycle of the ITCZ and $r_{y}$ is the e-folding length of the ITCZ function. The function $f(t)$ is prescribed as a half a sine during half a day and zero otherwise, repeating the cycle forever in order to simulate the diurnal cycle of the specific humidity in the ITCZ. In fact, recent studies have provided observational evidence of a significant diurnal variability in both the sea surface temperature (SST) (Bernie et al. 2005) and the convective activity along the ITCZs (Yang and Slingo 2001 and references therein). The function $f(t)$ may be Fourier expanded according to

$f(t)=\sum_{j \geq 0} f_{j} \cos \left(\frac{2 \pi j}{\tau} t\right)$ 
with

$f_{j}=\frac{2}{\pi\left(1-4 j^{2}\right)}$,

where $\tau$ is the duration of the day in dimensionless units. For $c=51 \mathrm{~m} \mathrm{~s}^{-1}$ and $\beta=2.3 \times 10^{-11} \mathrm{~m}^{-1} \mathrm{~s}^{-1}, T_{E} \approx 8.3 \mathrm{~h}$ and, consequently, $\tau \approx 2.9$.

To analyse the linear interaction between equatorial Rossby and inertio-gravity wave modes through the parametric forcing, let us consider a solution with only two modes, a Rossby mode having a zonal wavenumber $k_{R}$, meridional index $n_{R}$ and eigenfrequency $\omega_{R}$ and an inertio-gravity wave mode having a zonal wavenumber $k_{G}$, meridional index $n_{G}$ and eigenfrequency $\omega_{G}$, that is

$$
\begin{aligned}
{\left[\begin{array}{c}
V_{1}(x, y, t) \\
p_{1}(x, y, t)
\end{array}\right]=} & R(t)\left[\begin{array}{l}
u_{R}(y) \\
v_{R}(y) \\
p_{R}(y)
\end{array}\right] \mathrm{e}^{\mathrm{i} k_{R} x+\mathrm{i} \omega_{R} t} \\
& +G(t)\left[\begin{array}{c}
u_{G}(y) \\
v_{G}(y) \\
p_{G}(y)
\end{array}\right] \mathrm{e}^{\mathrm{i} k_{G} x+\mathrm{i} \omega_{G} t}+c . c,
\end{aligned}
$$

where $c . c$ indicates the complex conjugate of the previous term, $R(t)$ and $G(t)$ correspond to the spectral amplitudes of the Rossby and inertio-gravity modes, respectively; and the eigenfunctions with subscripts $R$ and $G$ are given by (5c) for $\omega_{a}=\omega_{R}, k=k_{R}$, $n=n_{R}$ and $\omega_{a}=\omega_{G}, k=k_{G}, n=n_{G}$, respectively. Substituting the ansatz (12) into (9) and (2a) and making use of (10) and (11) yield

$\frac{\mathrm{d} R}{\mathrm{~d} t}=\eta_{R} \Gamma f_{j} G^{*}$

$\frac{\mathrm{d} G}{\mathrm{~d} t}=\eta_{G} \Gamma f_{j} R^{*}$

provided the modes satisfy the following 'resonance relations' with the harmonics of the diurnal cycle of the ITCZ function

$k_{R}=k_{G}$

$\omega_{R}+\omega_{G} \pm \frac{2 \pi}{\tau} j \approx 0, \quad$ for any $j>0$.

In (13), the superscript $*$ denotes the complex conjugate and $\eta_{R}$ and $\eta_{G}$ represent the coupling constants and are given by

$$
\begin{aligned}
\eta_{R}= & \int_{-\infty}^{+\infty} p_{R}(y)\left[\mathrm{i} k_{G} u_{G}(y)+\frac{\mathrm{d} v_{G}(y)}{\mathrm{d} y}\right] \\
& \times Q_{0} \exp \left[-\left(\frac{y-y_{0}}{r_{y}}\right)^{2}\right] \mathrm{d} y \\
\eta_{G}= & \int_{-\infty}^{+\infty} p_{G}(y)\left[\mathrm{i} k_{R} u_{R}(y)+\frac{\mathrm{d} v_{R}(y)}{\mathrm{d} y}\right] \\
& \times Q_{0} \exp \left[-\left(\frac{y-y_{0}}{r_{y}}\right)^{2}\right] \mathrm{d} y .
\end{aligned}
$$

The linear interaction coefficients $\eta_{R}$ and $\eta_{G}$ represent a measure of the intrinsic coupling between the equatorial Rossby and Poincaré modes through the convective forcing. Physically, they indicate how far the heating produced by the convergence induced by the Poincaré (Rossby) mode projects onto the Rossby (Poincaré) mode. Using (5c), the coupling constants may alternatively be written as

$\eta_{R}=\mathrm{i} \omega_{G} \gamma_{R G}$

$\eta_{G}=\mathrm{i} \omega_{R} \gamma_{R G}$,

where

$\gamma_{\mathrm{RG}}=\int_{-\infty}^{+\infty} p_{\mathrm{R}}(y) p_{\mathrm{G}}(y) Q_{0} \exp \left[-\left(\frac{y-y_{0}}{r_{y}}\right)^{2}\right] \mathrm{d} y$.

To analyse the potentiality of an inertio-gravity wave mode to excite a Rossby mode in this linear interaction through the convective forcing, let us consider the case where the Rossby mode's amplitude is zero initially and the initial amplitude of the Poincare mode is represented by $|G(t=0)|=G_{0}$. In this situation, for the case where $\omega_{R} \omega_{G}<0$, the analytical solution of system (13) is given in terms of the modes' energy by

$E_{R}(t)=|R(t)|^{2}=G_{0}^{2}\left(\left|\frac{\omega_{G}}{\omega_{R}}\right|\right) \sin ^{2}(\lambda t)$

$E_{G}(t)=|G(t)|^{2}=G_{0}^{2} \cos ^{2}(\lambda t)$

with the frequency $\lambda$ of the energy modulation given by

$\lambda=\Gamma\left|f_{j}\right|\left|\gamma_{R G}\right|\left(\left|\omega_{R} \omega_{G}\right|\right)^{\frac{1}{2}}$.

The case described by (17)-(18) refers to the resonant duos composed of a Rossby mode and an eastward propagating inertiogravity wave mode, where the resonance condition (14b) is only satisfied if $\left|\omega_{G}\right|>2 \pi j / \tau$, for any $j>0$. For the resonant duos composed of a Rossby mode and a westward propagating inertiogravity wave mode we have $\omega_{R} \omega_{G}>0$ and the amplitudes of the modes grow unboundedly in time, being described by hyperbolic functions. This case may be regarded as a wave instability problem. Conversely, in the present paper we aim to build the linear wave interaction theory involving a convective resonance with the diurnal cycle and, consequently, the unstable case is beyond the scope of this paper. Thus, we focus our analysis here on the resonant duos composed of a Rossby and an eastward propagating Poincaré mode, whose coupling coefficients are of opposite signs and, consequently, whose energies are finite in time.

Table 1 shows the first harmonic frequencies associated with the time dependent part of the ITCZ function. As the $j=0$ harmonic has a zero time-frequency, resonance between equatorial Rossby and inertio-gravity modes through this harmonic is impossible. Nevertheless, since there exist inertio-gravity wave modes having eigenfrequencies close to the frequencies of the 
Table 1. First harmonic frequencies of the time-dependent part of the ITCZ function for $c=51 \mathrm{~m} \mathrm{~s}^{-1}$ and $\beta=2.3 \times 10^{-11} \mathrm{~m}^{-1} \mathrm{~s}^{-1}$, implying $\tau \approx 2.9$

\begin{tabular}{lccc}
\hline $\mathrm{j}$ & $2 \pi j / \tau$ & Period of oscillation & Fourier coefficients $=\frac{2}{\pi\left(1-4 j^{2}\right)}$ \\
\hline 0 & 0 & $\infty$ & 0.6366 \\
1 & 2.1233 & $1 \mathrm{~d}$ & -0.2122 \\
2 & 4.2467 & $12 \mathrm{~h}$ & -0.04244 \\
3 & 6.3699 & $8 \mathrm{~h}$ & -0.01819 \\
\hline
\end{tabular}

Table 2. Examples of nearly resonant duos of equatorial Rossby (RW) and eastward propagating inertio-gravity wave (EGW) modes that interact through the $1 \mathrm{~d}$ harmonic of the ITCZ function

\begin{tabular}{ccccccc}
\hline & RW & EGW & $\omega_{R}$ & \multicolumn{1}{c}{$\omega_{G}$} & $\gamma_{R G} \times 10^{-4}$ & $\sqrt{\left|\frac{\omega_{G}}{\omega_{R}}\right|}$ \\
\hline 1 & $(6,1)$ & $(6,1)$ & 0.2873 & -2.361 & 6.149 & 2.87 \\
2 & $(5,2)$ & $(5,1)$ & 0.185 & -2.2144 & 8.493 & 3.46 \\
3 & $(8,2)$ & $(8,0)$ & 0.2215 & -2.3088 & -9.297 & 3.23 \\
4 & $(8,1)$ & $(8,0)$ & 0.2915 & -2.3088 & 11.642 & 2.81 \\
\hline
\end{tabular}

Notes: The table shows, from left to right, the duo components, their respective eigenfrequencies and the coupling constant $\gamma_{R G}$ of the duo (see text for explanation), as well as the semi-major axis of the ellipse described by (17). The modes are characterized, from left to right, by the zonal wavenumber $\alpha$ and the meridional mode $n$. The interaction coefficients shown in this table were calculated for $y_{0}=1.1$ $\left(\approx 1650 \mathrm{Km}\right.$ or $\left.15^{\circ}\right)$ and $r_{y}=1.0(1500 \mathrm{Km})$.

transient harmonics $(j>0)$ of the ITCZ function, near resonances between slow tropical waves and fast eastward propagating inertio-gravity waves through the transient harmonics $(j \geq 1)$ of the diurnal forcing are allowed. Examples of such resonances are displayed in Table 2. Table 2 shows examples of nearly resonant duos of equatorial Rossby and eastward propagating Poincaré wave modes that resonate through the $j=1(1 \mathrm{~d})$ harmonic of the ITCZ function. The table shows the duo components, their respective eigenfrequencies and the interaction constants $\gamma_{R G}$ given by $(16 \mathrm{c})$ for $y_{0}=1.1\left(\approx 1650 \mathrm{Km}\right.$ or $\left.15^{\circ}\right)$ and $r_{y}=1.0(1500 \mathrm{Km})$. The interaction coefficient $\gamma_{R G}$ has been computed numerically from (16c) by using the Gauss-Hermite quadrature formula.

The resonant duos displayed in Table 2 are representative examples of the four possible resonances involving equatorial Rossby and Poincaré modes through the convective forcing in the present model. Duo 1 is a representative example of interaction in which both Rossby and Poincaré modes are symmetric (i.e. have an odd meridional index). In this case, the pressure fields of the modes have an even symmetry about the equator. Duo 3, on the other hand, illustrates an interaction involving antisymmetric (even meridional index) Rossby and Poincaré modes, where the pressure fields of the modes have an odd symmetry about the equator. Duo 2 is a representative example of interaction involving an antisymmetric Rossby and a symmetric inertiogravity wave mode, while in the last possible case represented by Duo 4, the Rossby mode is symmetric and the Poincare mode is antisymmetric.

For the resonant duos displayed in Table 2, eq. (17) describes an ellipse in phase space with semi-major axis $G_{0} \sqrt{\left|\frac{\omega_{G}}{\omega_{R}}\right|}$ and semi-minor axis given by $G_{0}$. Thus, as can be observed in Table 2, the maximum amplitude attained by the excited Rossby mode in this resonant interaction, which is given by the semimajor axis of the ellipse, is around three times the maximum (initial) amplitude of the inertio-gravity mode. Moreover, as the semi-major axis of the ellipse depends only on the ratio between the time-frequencies of the wave modes, the maximum amplitude of the excited Rossby mode is independent of the position and width of the ITCZ. The only exception, of course, occurs for Duos 2 and 4 when $y_{0}=0$. In this case, the ITCZ function is symmetric about the equator and, consequently, the integrand in (16c) has an odd symmetry about the equator and the interaction coefficient $\gamma_{R G}$ is zero.

Therefore, in the linear interaction mechanism involving the diurnal cycle of the convective forcing, the amplitude (energy) modulation of the equatorial Rossby mode is much stronger than the amplitude (energy) modulation of the inertio-gravity mode. In addition, as discussed in Section 1, such a significant energy modulation of the Rossby mode due to the interaction with an inertio-gravity mode is not possible in the context of the resonant non-linear triad interactions involving two inertiogravity waves and one Rossby mode. This restriction in the amplitude modulation of the Rossby modes in the resonant triad interactions involving two high-frequency modes is due to the energy constraints imposed by the triad dynamics, which force a Rossby mode to essentially act as a catalyst component for the energy exchanges between two inertio-gravity waves in a resonant triad. Therefore, the results presented in this section suggest that the diurnal cycle of tropical deep convection in the ITCZ can be a possible dynamical mechanism that leads a Rossby mode to be significantly excited by a fast-propagating inertio-gravity mode.

In order to investigate the potential relevance of the wave interaction theory developed here for the real atmosphere, it is also important to analyse how the displacement of the ITCZ, as well as its width, affects the strength of the interactions illustrated in Table 2. In this way, Figs. 2 and 3 show the interaction period given by $T_{\mathrm{int}}=2 \pi / \lambda$ (with $\lambda$ given by eq. 18) as a function of $y_{0}$ and $r_{y}$, respectively. In both cases, the height of the lift condensation level has been set with its standard value of $z_{m}=$ $2 \mathrm{Km}$. This value is typical over most of the equatorial oceans. In Fig. 2 the parameter $r_{y}$ is fixed as $r_{y}=1.0$, whereas in Fig. 3 the latitude of the forcing is fixed as $y_{0}=1.0(1500 \mathrm{Km})$. The interaction period $T_{\text {int }}$ is a measure of the strength of the energy exchanges between the Rossby and Poincare modes, since the stronger the interaction, the shorter the interaction period. 

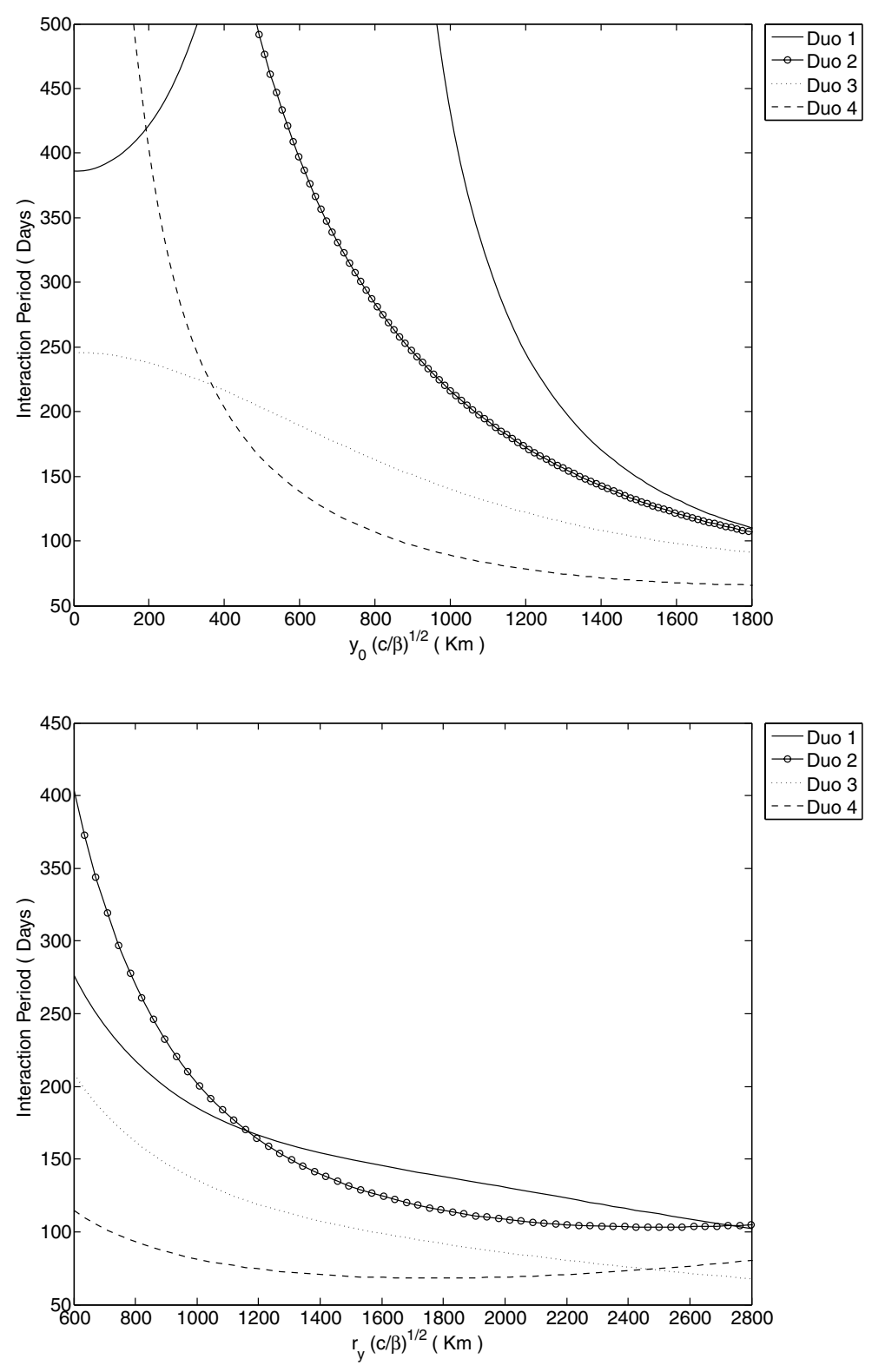

Fig. 2. Interaction period $T_{\text {int }}=2 \pi / \lambda$ (with $\lambda$ given by eq. 18) as a function of $y_{0}$ for $r_{y}=1.0(\approx 1500 \mathrm{Km})$.
Figure 2 shows that, in general, all the types of resonant duos undergo more expressive interactions when the ITCZ gets its maximum latitudinal displacement. In the case of Duo 1 where both Rossby and Poincaré wave modes have the $n=1$ meridional mode (symmetric about the equator), the strength of the interaction decreases as the ITCZ moves away from the equator such that the interaction becomes very weak as the ITCZ approaches the correspondent latitude of $y_{0}=0.5 L_{E}$. Then the interaction intensifies again and becomes more expressive as the ITCZ approaches $y_{0}=1.1\left(\approx 1650 \mathrm{Km}\right.$ or $\left.15^{\circ}\right)$, which corresponds to the maximum latitudinal displacement of the ITCZ in the Northern Hemisphere. For Duos 2 and 4, the interaction is zero when $y_{0}=0$ and, consequently, the interaction period becomes extremely long as the forcing approaches the equator.
Another point to be noted in Fig. 2 is that for Duo 1 the strongest interactions are associated with interaction periods of the order of $120 \mathrm{~d}$. This means that when this interaction is very active (i.e. when $y_{0}=1.1 L_{E}$ ), the Rossby mode attains its maximum amplitude at $t \approx 60 \mathrm{~d}$. Similarly, when the interactions associated with Duos 2, 3 and 4 are more active (i.e. when the ITCZ is away from the equator), the energy (amplitude) modulations occur in an intraseasonal time-scale (periods of the order of 60-120 d). As regards the dependence of the strength of the interactions upon the width of the ITCZ, Fig. 3 shows that, in general, the interactions are more intense for wider ITCZs, with the most marked variation occurring for the interaction involving an antisymmetric Rossby mode and a symmetric Poincaré wave mode (Duo 2). For Duo 4, which undergoes the strongest 
interaction, the variation of the strength of the interaction with the width of the ITCZ is very small. For narrow ITCZs ( $r_{y}$ ranging from 600 to around $1000 \mathrm{Km}$ ), the only interaction occurring on intraseasonal time-scale is that associated with Duo 4.

\subsection{Augmented two-wave interaction theory}

So far we have developed the two-wave interaction theory in which equatorial Rossby and eastward propagating inertiogravity wave modes can interact and exchange energy with each other through resonance with the diurnal cycle of the ITCZ function in the context of the heat forcing parametrization. However, recall that in the non-linear context of (1)-(2), the modes involved in this interaction may also participate of resonant triad non-linear interactions with the barotropic wave modes, so that in the full dynamics these linear resonances might somehow compete with the resonant triad non-linear interactions.

Thus, in order to illustrate in a simplified fashion on how the linear resonance mechanism involving equatorial Rossby and Poincaré wave modes through the diurnal forcing works in the full dynamics, where it may compete with the three-wave nonlinear resonances, we have also analysed in this work the reduced dynamics of a resonant wave triad coupled with a resonant duo through one of its wave components. In this way, let us consider a solution with four modes, namely a barotropic non-divergent wave mode having zonal wavenumber $k_{B}$, meridional wavenumber $l_{B}$ and eigenfrequency $\omega_{B}$ given by (7) for $k=k_{B}$ and $l=l_{B}$, two equatorial inertio-gravity wave modes constituting a nearly resonant triad with the barotropic wave mode, one with zonal wavenumber $k_{a}$, meridional index $n_{a}$ and eigenfrequency $\omega_{a}$ and the other with zonal wavenumber $k_{c}$, meridional index $n_{c}$ and eigenfrequency $\omega_{c}$, and an equatorial Rossby mode with zonal wavenumber $k_{R}$, meridional index $n_{R}$ and eigenfrequency $\omega_{R}$ interacting with one of the equatorial Poincare modes through the diurnal forcing. In other words, let us consider the following ansatz

$$
\begin{gathered}
\psi(x, y, t)=B(t) \sin \left(l_{B} y\right) \mathrm{e}^{\mathrm{i} k_{B} x+\mathrm{i} \omega_{B} t}+c . c \\
{\left[\begin{array}{c}
u_{1}(x, y, t) \\
v_{1}(x, y, t) \\
p_{1}(x, y, t)
\end{array}\right]=R(t)\left[\begin{array}{l}
u_{R}(y) \\
v_{R}(y) \\
p_{R}(y)
\end{array}\right] \mathrm{e}^{\mathrm{i} k_{R} x+\mathrm{i} \omega_{R} t}} \\
+G_{a}(t)\left[\begin{array}{c}
u_{a}(y) \\
v_{a}(y) \\
p_{a}(y)
\end{array}\right] \mathrm{e}^{\mathrm{i} k_{a} x+\mathrm{i} \omega_{a} t} \\
+G_{c}(t)\left[\begin{array}{c}
u_{c}(y) \\
v_{c}(y) \\
p_{c}(y)
\end{array}\right] \mathrm{e}^{\mathrm{i} k_{c} x+\mathrm{i} \omega_{c} t}+c . c
\end{gathered}
$$

with the following relations satisfied

$$
k_{a} \pm k_{\mathrm{B}}=k_{c}
$$

$n_{a}+n_{c}=$ even

$\omega_{B} \pm \omega_{a} \pm \omega_{c} \approx 0$

and

$k_{R}=k_{a}$

$\omega_{R}+\omega_{a} \approx \frac{2 \pi}{\tau}$

Then, inserting the ansatz (19) into (4), (2a) and (9) and making use of (20), (21) and (10)-(11) yield

$\frac{\mathrm{d} B}{\mathrm{~d} t}=\sigma_{B} G_{a}^{*} G_{c}$

$\frac{\mathrm{d} G_{a}}{\mathrm{~d} t}=\sigma_{a} B^{*} G_{c}+\eta_{G} f_{j} \Gamma R^{*}$

$\frac{\mathrm{d} G_{c}}{\mathrm{~d} t}=\sigma_{c} B G_{a}$

$\frac{\mathrm{d} R}{\mathrm{~d} t}=\eta_{R} \Gamma f_{j} G_{a}^{*}$.

In (22), $\eta_{R}$ and $\eta_{G}$ are given by (15) or (16) and the non-linear coupling coefficients $\sigma_{B}, \sigma_{a}$ and $\sigma_{c}$ are written as

$$
\begin{aligned}
\sigma_{B}= & \frac{1}{k_{B}^{2}+l_{B}^{2}} \int_{-\infty}^{+\infty} \sin \left(l_{B} y\right)\left[u_{a} \mathrm{i} k_{c} \zeta_{c}+v_{a} \frac{\mathrm{d} \zeta_{c}}{\mathrm{~d} y}+2 \zeta_{a} \operatorname{div}_{i k} V_{c}\right. \\
& \left.-V_{a}^{\perp} \bullet\left(-k_{c}^{2} u_{c}+\mathrm{i} k_{c} \frac{\mathrm{dv}_{c}}{\mathrm{~d} y}, \mathrm{i} k_{c} \frac{\mathrm{d} u_{c}}{\mathrm{~d} y}+\frac{d^{2} \mathrm{v}_{c}}{\mathrm{~d} y^{2}}\right)^{T}+C P\right] \mathrm{d} y
\end{aligned}
$$

$$
\begin{aligned}
\sigma_{a}= & -\int_{-\infty}^{+\infty}\left[u_{B} \mathrm{i} k_{c} u_{c}+v_{B} \frac{d u_{c}}{d y}+C P\right] u_{a}(y) \mathrm{d} y \\
& -\int_{-\infty}^{+\infty}\left[u_{\mathrm{B}} \mathrm{i} k_{c} v_{c}+v_{\mathrm{B}} \frac{\mathrm{dv}_{c}}{\mathrm{~d} y}+C P\right] \mathrm{v}_{a}^{*}(y) \mathrm{d} y \\
& -\int_{-\infty}^{+\infty}\left[u_{B} \mathrm{i} k_{c} \mathrm{p}_{c}+v_{B} \frac{\mathrm{dp}_{c}}{\mathrm{~d} y}\right] p_{a}(y) \mathrm{d} y .
\end{aligned}
$$

In (23) above, $u_{B}(y)=-l_{B} \cos \left(l_{B} y\right), v_{B}(y)=\mathrm{i} k_{B} \sin \left(l_{B} y\right)$ correspond to the velocity field of the barotropic eigenmode, $\operatorname{div}_{i k}$ $V_{j}(y)=\mathrm{i} k_{j} u_{j}(y)+\mathrm{d} v_{j}(y) / \mathrm{d} y$, for $j=a$ or $c$, refers to the divergence of the equatorial eigenmodes, while $\varsigma_{j}=\mathrm{i} k_{j} v_{j}(y)-\mathrm{d} u_{j}(y) / \mathrm{d} y$, for $j=a$ or $c$, is their relative vorticity; $V_{j}(y)=\left[u_{j}(y), v_{j}(y)\right]^{T}$, for $j=a$ or $c$, corresponds to the velocity field of the equatorial eigenmodes, whereas $V_{j}^{\perp}(y)=\left(-\mathrm{v}_{j}, u_{j}\right)^{T}$. The term CP in (23) represents the cyclical permutation of what is proceeding, and the non-linear coupling coefficient $\sigma_{c}$ is written exactly as in (23b) but exchanging the subscripts a and c. Physically, the interaction coefficient $\sigma_{B}$ represents a measure of how far the vorticity transport produced by the two equatorial Poincaré wave modes projects onto the barotropic mode. Conversely, the non-linear coupling coefficients $\sigma_{a}$ and $\sigma_{c}$ represent a measure of how far the advection of momentum of one of the equatorial 
wave modes produced by the barotropic mode and the advection of momentum of the barotropic mode produced by one of the equatorial modes, as well as the advection of the mass field of the equatorial wave mode produced by the barotropic wind field, project onto the other equatorial eigenmode. The non-linear interaction coefficients $\sigma_{a}, \sigma_{c}$ and $\sigma_{B}$ have also been computed numerically by using the Gauss-Hermite quadrature formula. The relations (20a) and (20c) correspond to the usual non-linear resonant triad interaction conditions, while condition (20b) is necessary for the integrand in (23) to have an even symmetry about the equator. In case of an odd symmetry, the resonant component generated in $y \geq 0$ would be exactly cancelled by the resonant component generated in $y \leq 0$.

We have then numerically integrated the reduced dynamical eqs (22) for the system composed of a barotropic wave mode having zonal wavenumber $1(\alpha=1)$ and the second meridional wavenumber, and two eastward propagating inertio-gravity wave modes having meridional mode $n=0$ : one with zonal wavenum- ber 8 and the other with zonal wavenumber 7 . The equatorial Rossby mode in this case is that with zonal wavenumber 8 and meridional index $n=1$. Note that the resonant duo here is the Duo 4 of Table 2, which undergoes the most significant interaction through the diurnal forcing. For the numerical integration we have used the Matsuno (predictor-corrector) scheme at the first step with a time-step of $\Delta t / 6$ and the Leapfrog scheme at the remaining integration steps with a time-step of $\Delta t$. In most of our numerical integrations we have set $\Delta t=10 \mathrm{~min}$.

Results of numerical integrations of system (22) are shown in Fig. 4 for the initial condition defined in such a way that the initial energy is almost totally projected onto mode $a$, with just an infinitesimally small part of total energy being projected onto the remaining modes of the resonant triad in a white noise form. Figure $4 \mathrm{a}$ shows the result of a numerical simulation for the case where the heat forcing is centred at the equator $\left(y_{0}=0\right)$, whereas Figs $4 \mathrm{~b}$ and $\mathrm{c}$ display results of numerical simulations for $y_{0}=0.2\left(\approx 300 \mathrm{Km}\right.$ or $\left.3^{\circ}\right)$ and $y_{0}=1.1\left(\approx 1650 \mathrm{Km}\right.$ or $\left.15^{\circ}\right)$, (a)

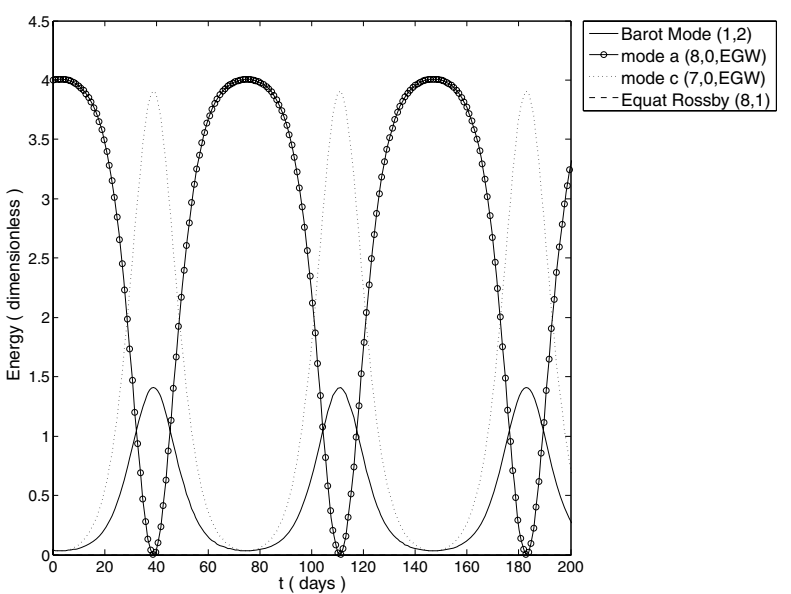

(b)

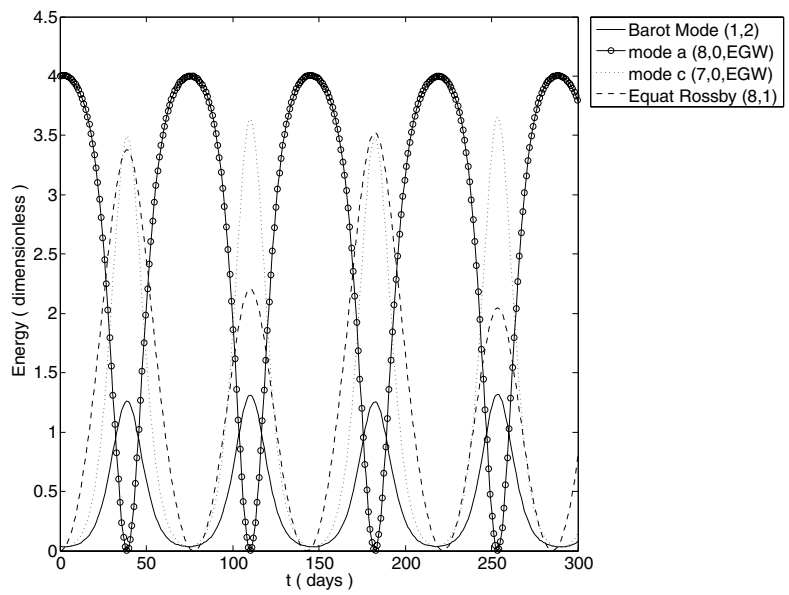

(c)

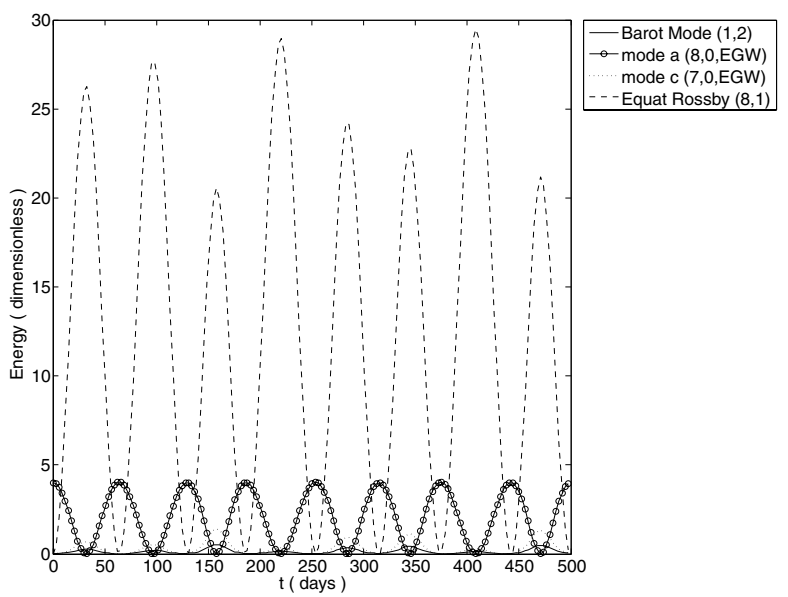

Fig. 4. Time evolution of the modes' energy associated with the numerical solution of (22) for the initial amplitudes given by $|B(t=0)|=0.2$, $\left|G_{a}(\mathrm{t}=0)\right|=2.0,\left|G_{c}(\mathrm{t}=0)\right|=0.2$ and $|R(t=0)|=0.0$. In the numerical simulations illustrated here, the width of the ITCZ is fixed with $r_{y}=1.0$ $(\approx 1500 \mathrm{Km})$ and the latitude of the ITCZ is set as: (a) $y_{0}=0.0$, (b) $y_{0}=0.2(\approx 300 \mathrm{Km})$ and $(\mathrm{c}) y_{0}=1.1(\approx 1650 \mathrm{Km})$. The energy of the barotropic eigenmode is given by $\left(k_{B}^{2}+l_{B}^{2}\right)|B(t)|^{2}$, while the energy of the equatorial wave modes is given by the square of their amplitudes. 
respectively. In all the simulations displayed in Fig. 4 the width of the ITCZ is fixed with $r_{y}=1.0(1500 \mathrm{Km})$. In these simulations, the initial amplitude (energy) of the equatorial Rossby mode is zero.

This scenario where only one wave mode holds almost total initial energy may be regarded as a wave stability problem. In the context of the non-linear triad interaction, it is well known that a single wave mode is unstable only if it is the highest absolute frequency mode of the triad. In this case, the mode containing the most part of the initial energy loses its energy to the other two triad components until its energy gets small enough for the constraints imposed by the total energy conservation to yield saturation for the energy of the two remaining modes of the triad. After this energy level, the highest frequency mode of the resonant triad begins receiving energy from the other waves until it becomes unstable and the cycle repeats, with the energy of the highest absolute frequency mode always growing or decaying at the expense of the remaining triad components. This is exactly what happens in the simulation displayed in Fig. 4a. In this case $\left(y_{0}=0\right)$, the ITCZ function is symmetric about the equator and the linear interaction coefficient associated with Duo 4 of Table 2 is zero. As a consequence, eq. (22b) decouples from eq. (22d), and (22) reduces to the dynamics of a single resonant wave triad. The energy exchanges observed in Fig. 4a constitute a typical picture of a resonant triad non-linear interaction involving two inertio-gravity wave modes and a Rossby mode, where the energy modulation of the Rossby mode is smaller than the energy modulations of the two high frequency modes. The role of the low-frequency mode in this case is essentially to act as a catalyst mode for the energy exchange between the two inertio-gravity waves.

Figure $4 b$ illustrates the energy modulations of the modes when the linear interaction between the inertio-gravity mode and the equatorial Rossby mode of (22) is of moderate level, that is, when the ITCZ is slightly moved away from the equator. In this case, as can be noted in Fig. 4b, apart from the energy growth of the other triad components, the duo interaction between the unstable inertio-gravity wave mode and the equatorial Rossby mode leads to the excitation of the latter. Nevertheless, as the non-linear interaction is stronger than the linear one in this case, the excited equatorial Rossby mode no longer attains its maximum amplitude level predicted by the linear theory described by (17). Instead, the competition between the duo and the triad interactions makes the energy of the equatorial Rossby mode to decrease earlier than predicted by the two-wave interaction theory, so that the equatorial Rossby mode undergoes in this case an amplitude modulation with a period of $70 \mathrm{~d}$, which is much shorter than predicted by the linear theory for these values of $y_{0}$ and $r_{y}$ (Fig. 2). Another interesting feature to be observed in Fig. $4 \mathrm{~b}$ is that the competition between the linear and non-linear interactions makes the energy of the equatorial Rossby mode to go through a period-doubling energy modulation, alternating small and large energy peaks within a total oscillation period of the order of $140 \mathrm{~d}$.

However, besides this energy modulation with periods of the order of 70 and 140 d, Fig. 5 shows that the competition between the two-wave and the three-wave interactions yields an even longer time-scale modulation in the modes' energies. Figure 5 displays the result of the same numerical simulation of Fig. $4 \mathrm{~b}$ but for very longer time integration. Fig. 5 illustrates the time evolution of the total energy of the triad, given by the sum of the energies of the barotropic and the two equatorial inertio-gravity modes, and the time evolution of the energy of the equatorial Rossby mode. Fig. 5 illustrates that, in a very long time-scale, the energy of the equatorial Rossby mode grows significantly and attains its maximum level predicted by the two-wave theory
Fig. 5. Time evolution of the energy of the equatorial Rossby mode $\left(|R(t)|^{2}\right)$ and the total energy of the triad, given by $\left(k_{B}^{2}+l_{B}^{2}\right)|B(t)|^{2}+\left|G_{a}(t)\right|^{2}+\left|G_{c}(t)\right|^{2}$, for the same numerical solution of Fig. $4 \mathrm{~b}$.

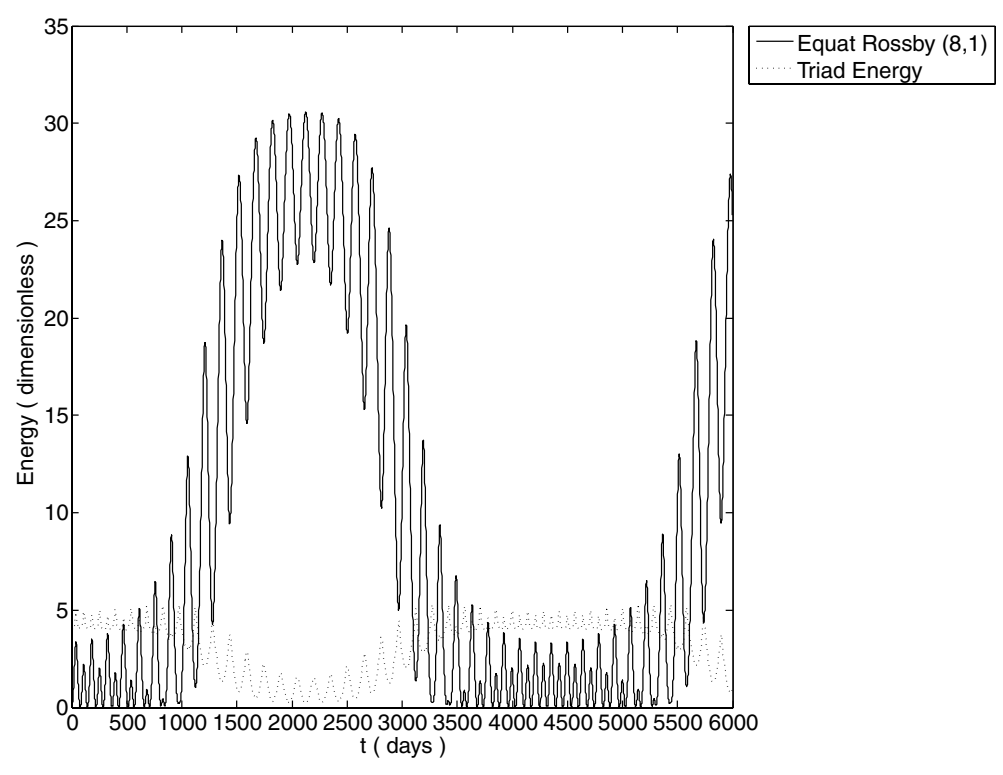


(17), stealing energy from the triad. Thus, one notices in Fig. 5 that, as a result of the competition between the non-linear triad and the linear duo interactions, the equatorial Rossby mode and the wave triad exchange energy in a very long time-scale, apart from undergoing relatively small amplitude energy modulations in intraseasonal and semi-annual time-scales.

For $y_{0}=1.1$, which roughly corresponds to the maximum latitudinal displacement of the ITCZ, the linear interaction between the equatorial Rossby and the inertio-gravity modes gets its strongest level (Fig. 2). In this case, Fig. 4c shows that the excitation of the barotropic and the zonal wavenumber 7 inertiogravity wave modes is very inhibited, and the energy of the unstable Poincaré mode almost entirely leaks to the equatorial Rossby mode. Like in the reduced dynamics of a single resonant duo, the zonal wavenumber 8 equatorial Rossby mode with meridional index $n=1$ undergoes an expressive energy modulation due to the interaction with the equatorial eastward propagating inertio-gravity wave with zonal wavenumber 8 and meridional index $n=0$. In addition, the period of the energy modulation of the equatorial Rossby mode observed in Fig. 4c $(65 \mathrm{~d})$ is close to that predicted by the two-wave theory for $y_{0}=$ $1.1 L_{E}$ and $r_{y}=1.0 L_{E}$ (Fig. 2). Thus, in the example illustrated in Fig. 4c, the linear interaction through the diurnal forcing is more effective and, consequently, overcomes the non-linear triad energy exchanges.

\section{Concluding remarks}

In this work, we have presented a linear wave interaction theory in which equatorial Rossby and eastward propagating inertiogravity wave modes may interact and exchange energy with each other through latitudinal and time inhomogeneities of the background distribution of moisture in the tropical atmosphere, in the context of deep convection parametrization. The latitudinal inhomogeneity is associated with the structure of the ITCZ, whereas the time inhomogeneity is related to the diurnal variation of tropical deep convection. We have adopted the two-layer incompressible equatorial primitive equations forced by a parametric heating that roughly represents deep convection activity in the tropical atmosphere. The heat source was parametrized in the simplest way according to the hypothesis that it is proportional to the lower-troposphere moisture convergence, with the background moisture state function mimicking the structure of the ITCZ. In this context, we have investigated the possibility of resonant interaction between equatorially trapped Rossby and inertio-gravity modes through the diurnal cycle of the background moisture state function.

The reduced dynamics of a single resonant duo shows that the linear interaction between equatorial Rossby and eastward propagating Poincaré modes through the fixed diurnal forcing can be described by the parametric equations of an ellipse in phasespace, with the semi-major axis representing the amplitude of the equatorial Rossby mode and the semi-minor axis describing the amplitude of the equatorial Poincaré mode. According to the theory we present, the semi-major axis of the ellipse depends only on the ratio between the two eigenfrequencies so that the amplitude of the excited Rossby mode does not depend on the latitudinal displacement of the ITCZ. The only exception occurs for the resonant duos involving symmetric and antisymmetric modes when the ITCZ function is centred at the equator. In this case, the wave interaction is zero and a Rossby mode cannot be excited by an existing Poincaré mode.

Therefore, in this linear interaction mechanism involving the diurnal cycle of the convective forcing, the amplitude (energy) modulation of the equatorial Rossby mode is much stronger than the amplitude (energy) modulation of the inertio-gravity mode. In addition, as discussed in Section 1, such a significant energy modulation of the Rossby mode due to the interaction with an inertio-gravity mode is not possible in the context of the resonant non-linear triad interactions involving two inertiogravity waves and one Rossby mode. This restriction in the amplitude modulation of the Rossby modes in the resonant triad interactions involving two high-frequency modes is due to the energy constraints imposed by the triad dynamics, which force a Rossby mode to essentially act as a catalyst component for the energy exchanges between two inertio-gravity waves. Therefore, the theory we present here suggests that the diurnal cycle of tropical deep convection in the ITCZ can be a possible dynamical mechanism that leads a Rossby mode to be significantly excited by a fast-propagating inertio-gravity mode.

The results of the two-wave dynamics also point out that this linear interaction mechanism involving equatorial Rossby and Poincaré modes through the diurnal forcing is more effective when the ITCZ is located away from the equator, which is characteristic of extreme seasons (summer and winter). For the ITCZ function centred nearby the equator, which is characteristic of transition seasons, the only significant interaction is that associated with Duo 3 of Table 2, which involves two antisymmetric modes. Another point regarding the linear wave interaction theory developed here is that when the duo interactions are very active, that is, when the ITCZ is located away from the equator, the modes undergo amplitude modulations at intraseasonal time-scales (periods of the order of 60-120 d). As regards the dependence of the strength of the interaction upon the width of the ITCZ, the results show that, in general, the interactions are more intense for wider ITCZs. For narrow ITCZs $\left(r_{y}\right.$ ranging from 600 to around $1000 \mathrm{Km}$ ), the only interaction occurring on intraseasonal time-scale is that associated with Duo 4, which involves a symmetric Rossby mode and an antisymmetric inertio-gravity wave mode.

In order to illustrate in a simplified fashion on how this resonance mechanism works in the full dynamics, where it may compete with the three-wave non-linear resonance, we also augment the two-wave interaction theory to include the three-wave nonlinear interaction of equatorial inertio-gravity wave modes and a barotropic mode. In this sense, we have analysed the dynamics 
of a resonant non-linear wave triad composed of two equatorial eastward propagating inertio-gravity waves and one barotropic wave mode in which the highest absolute frequency Poincaré mode interacts with an equatorial Rossby mode through the diurnal forcing. We have focused on the case where the highest absolute frequency Poincaré mode holds most part of the initial energy. The numerical results of the four-wave dynamics show that when the duo interaction is very active, that is, when the ITCZ is located away from the equator, the excitation of the equatorial Rossby mode overcomes the triad non-linear energy exchanges, and the linear interaction involving the diurnal forcing dominates the system's dynamics. On the other hand, when the linear interaction between the Poincaré and the equatorial Rossby modes is of moderate level, that is, when the ITCZ is slightly displaced from the equator, the competition between the non-linear triad and the linear duo interactions leads the equatorial Rossby mode and the wave triad to exchange energy in a very long time-scale. In addition, besides this ultralong timescale energy exchange, both the equatorial Rossby mode and the triad components undergo amplitude (energy) modulations in intraseasonal and semi-annual time-scales.

Therefore, the results presented in this paper also have important implications for the long-term weather forecast. The results suggest that the correct representation of the multi time-scale interaction presented here relies on the correct representation in numerical weather forecast models of the diurnal cycle of the convective activity over the ITCZs. Furthermore, the results suggest that the diurnal cycle of the ITCZ might be important for generating low-frequency (intraseasonal, semi-annual and even longer term) fluctuations in the atmospheric circulation. In addition, in a more complete picture of the model (1)-(2), an equatorial Rossby mode excited by an equatorial Poincaré wave mode through the diurnal forcing may also non-linearly interact and exchange energy with barotropic Rossby modes (Majda and Biello, 2003; Biello and Majda, 2004a,b; Reznik and Zeitlin, 2006; 2007). This kind of interaction involving equatorial baroclinic and barotropic Rossby modes, in the presence of the parametric diurnal forcing considered here, might generate a tropical-extratropical connection directly related to diurnal variations of deep convection in the tropics.

An important point in the construction of the wave interaction theory developed here refers to the convective parametrization, which is still an open problem in atmospheric sciences. In the present work we have considered the simplest convective parametrization based on the wave-CISK hypothesis in which the heating is assumed to be proportional to the lower-troposphere moisture convergence. This choice was made because of the simplicity in exposition associated with this parametrization, which requires just a single first baroclinic vertical heating profile. However, this concept of a single deep convection mode parametrization constitutes an oversimplified model for describing synoptic and planetary-scale convectively coupled equatorial waves, in the sense that recent observational studies (e.g. Lin and Johnson, 1996; Johnson et al., 1999) have identified the important role of both the stratiform and the congestus heating in the life-cycles of these tropical systems. This multicloud nature of tropical convection led to the design of models for convective parametrization carrying the first and the second baroclinic modes (e.g. Khouider and Majda, 2006; 2007; 2008; Mapes, 2000; Majda et al., 2007).

Nevertheless, in spite of the oversimplified convective parametrization considered in the present work, the theory developed here can be generalized to more complex parametrizations. For instance, it is possible to realize that the effect of adding the second baroclinic mode in our analysis in order to take into account the multicloud nature of tropical convection would be simply to spread the possibility of duo interactions between equatorial Rossby and Poincaré wave modes. Thus, the theory presented here would be very similar if we considered a multicloud convective parametrization, but with more possibilities of resonances. Consequently, in a more complex model the resonances associated with the diurnal cycle of tropical convection might be even more favoured than indicated in this simplified context.

\section{Acknowledgments}

The work of Carlos F. M. Raupp was supported by FAPESP (Fundação de Amparo à Pesquisa do Estado de São Paulo) through grants 06/53606-0 and 09/11643-4. Pedro L. Silva Dias is supported by grants from PROSUR/IRI and Moore Foundation. We would also like to thank the anonymous reviewers for their useful suggestions to improve this manuscript.

\section{References}

Baer, F. and Tribbia, J. J. 1977. On the complete filtering of gravity modes through nonlinear initialization. Mon. Wea. Rev. 105, 1536-1539.

Bernie, D. J., Woolnough, S. J., Slingo, J. M. and Guilyardi, E. 2005. Modeling diurnal and intraseasonal variability of the ocean mixed layer. J. Climate 18, 1190-1202.

Biello, J. A. and Majda, A. J. 2004a. Boundary layer dissipation and the nonlinear interaction of equatorial baroclinic and barotropic Rossby waves. Geophys. Astrophys. Fluid Dyn. 98(2), 85-127.

Biello, J. A. and Majda, A. J. 2004b. The effect of meridional and vertical shear on the interaction of equatorial baroclinic and barotropic Rossby waves. Stud. Appl. Math. 112, 341-390.

Bretherton, F. P. 1964. Resonant interactions between waves. The case of discrete oscillations. J. Fluid. Mech. 20, 457-479.

Chang, C.-P. and Lim, H. 1988. Kelvin wave-CISK: a possible mechanism for the 30-50 day oscillations?. J. Atmos. Sci. 45, 1709-1720.

Charney, J. 1955. The use of the primitive equations of motion in numerical prediction. Tellus 7, 22-26.

Craik, A. D. D. 1985. Wave Interactions and Fluid Flows. Cambridge Monographs on Mechanics and Applied Math. Cambridge University Press, New York, pp. 322.

Duffy, D. G. 1974. Resonant interactions of inertio-gravity and Rossby waves. J. Atmos. Sci., 31, 1218-1231. 
Domaracki, A. and Loesch, A. Z. 1977. Nonlinear interactions among equatorial waves. J. Atmos. Sci. 34, 486-498.

Hayashi, Y. 1970. A theory of large-scale equatorial waves generated by condensational heat and accelerating the zonal wind. J. Meteor. Soc. Japan 48, 140-160.

Johnson, R. H., Rickenbach, T. M., Rutledge, S. A., Ciesielski, P. E. and Schubert, W. H. 1999. Trimodal characteristics of tropical convection. J. Climate 12, 2397-2407.

Kasahara, A. 1982. Nonlinear normal mode initialization and the bounded derivative method. Rev. Geophys. Space Phys. 20, 385-397.

Khouider, B. and Majda, A. J. 2006. A simple multicloud parameterization for convectively coupled tropical waves. Part I: linear analysis. $J$. Atmos. Sci. 63, 1308-1323.

Khouider, B. and Majda, A. J. 2007. A simple multicloud parameterization for convectively coupled tropical waves. Part II: nonlinear simulations. J. Atmos. Sci. 64, 381-400.

Khouider, B. and Majda, A. J. 2008. Multicloud models for organized tropical convection: enhanced congestus heating. J. Atmos. Sci. 65 , 895-914.

Lin, X. and Johnson, R. H. 1996. Kinematic and thermodynamic characteristics of the flow over the western Pacific warm pool during TOGA COARE. J. Atmos. Sci. 53, 695-715.

Lindzen, R. S. 1974. Wave-CISK in the tropics. J. Atmos. Sci. 31, 156-179.

Loesch, A. Z. and Deininger, R. C. 1979. Dynamics of closed systems of resonantly interacting equatorial waves. J. Atmos. Sci. 36, 1490-1497.

Majda, A. J. and Biello, J. A. 2003. The nonlinear interaction of barotropic and equatorial baroclinic Rossby waves. J. Atmos. Sci., 60, 1809-1821.

Majda, A. J. and Embid, P. 1998. Averaging over fast waves for geophysical flows with unbalanced initial data. Theor. Comput. Fluid Dyn. 11, 155-169.

Majda, A. J., Rosales, R. R., Tabak, E. G. and Turner, C. V. 1999. Interaction of large-scale equatorial waves and dispersion of Kelvin waves through topographic resonances. J. Atmos. Sci. 56, 4118-4133.

Majda, A. J., Stechmann, S. and Khouider, B. 2007. Madden-Julian oscillation analog and intraseasonal variability in a multicloud model above the equator. PNAS (USA) 104(24), 9919-9924.

Mapes, B. E. 2000. Convective inhibition, subgrid-scale triggering energy, and stratiform instability in a toy tropical wave model. J Atmos. Sci. 57, 1515-1535.

Matsuno, T. 1966. Quasi-geostrophic motions in the equatorial area. $J$. Meteor. Soc. Japan 44, 25-43.
Medvedev, S. B. and Zeitlin, V. 2005. Weak turbulence of short equatorial waves. Phys. Lett. A 342, 217-227.

Milewski, P. 1995. Resonant interactions between vortical flows and water waves. Part II: shallow water. Stud. Appl. Math. 94, 225256.

Milewski, P. 1998. Long wave interaction over varying topography. Physica D 123, 36-47.

Milewski, P. and D. J. Benney. 1995. Resonant interactions between vortical flows and water waves. Part I: deep water. Stud. Appl. Math. 94, 131-167.

Phillips, N. A. 1960. On the problem of initial data for the primitive equations. Tellus 12, 121-126.

Raupp, C. F. M. and Silva Dias, P. L. 2006. Dynamics of resonantly interacting equatorial waves. Tellus 58A, 263-279.

Raupp, C. F. M., Silva Dias, P. L., Tabak, E. G. and Milewski, P. 2008. Resonant wave interactions in the equatorial wave-guide. J. Atmos. Sci. 65, 3398-3418.

Reznik, G. M. and Zeitlin, V. 2006. Resonant excitation of Rossby waves in the equatorial waveguide and their nonlinear evolution. Phys. Rev. Lett. 96, 034502.

Reznik, G. M. and Zeitlin, V. 2007. Interaction of free Rossby waves with semi-transparent equatorial waveguide. Part 1. Wave triads. Physica D 226, 55-79.

Ripa, P. 1981. On the theory of nonlinear interactions among geophysical waves. J. Fluid Mech. 103, 87-115.

Ripa, P. 1982. Nonlinear wave-wave interactions in a One-Layer Reduced-Gravity Model on the equatorial $\beta$-plane. J. Phys. Oceanogr. 12, 97-111.

Ripa, P. 1983a. Weak interactions of equatorial waves in a one-layer model. Part I: general properties. J. Phys. Oceanogr. 13, 1208-121226.

Ripa, P. 1983b. Weak interactions of equatorial waves in a one-layer model. Part II: applications. J. Phys. Oceanogr. 13, 1227-121240.

Silva Dias, P. L., Schubert, W. H. and DeMaria, M. 1983. Large-scale response of the tropical atmosphere to transient convection. J. Atmos. Sci. 40, 2689-2707.

Stevens, D. E. and Lindzen, R. S. 1978. Tropical wave-CISK with a moisture budget and cumulus friction. J. Atmos. Sci. 35, 940-961.

Tribbia, J. J. 1979. Non-linear initialization on an equatorial beta-plane. Mon. Wea. Rev. 107, 704-713.

Tribbia, J. J. 1984. A simple scheme for high-order nonlinear normal mode initialization. Mon. Wea. Rev. 112, 278-284.

Yang, G-Y. and Slingo, J. 2001. The diurnal cycle in the tropics. Mon. Wea. Rev. 129, 784-801. 